\title{
Detection of Concealed Individuals Based on Their Vital Signs by Using a See-Through-Wall Imaging System With a Self-Injection-Locked Radar
}

\author{
Fu-Kang Wang, Student Member, IEEE, Tzyy-Sheng Horng, Senior Member, IEEE, \\ Kang-Chun Peng, Member, IEEE, Je-Kuan Jau, \\ Jian-Yu Li, Student Member, IEEE, and Cheng-Chung Chen
}

\begin{abstract}
This paper presents a self-injection-locked (SIL) radar with ranging and tracking capabilities to see through walls for discovering hidden people. Characterized by low complexity and high sensitivity, the proposed SIL radar merges the frequency-modulated continuous-wave and sum-difference pattern detection approaches to determine the distance and azimuth from the radar to each individual in a scene behind a wooden partition wall. An individual can be distinguished from a stationary object by using dynamic spectral subtraction to extract human motions or vital signs. Additionally, two or more individuals can be distinguished from each other by decomposing the Doppler signal into contributions of individuals in a polar domain. Consequently, a see-through-wall imaging system is constructed with the proposed SIL radar to position different individuals concealed behind a wall.
\end{abstract}

Index Terms-Frequency-modulated continuous-wave (FMCW) radar, see-through-wall (STW) imaging radar, self-injection-locked (SIL) radar, sum-difference pattern, vital signs.

\section{INTRODUCTION}

$\mathbf{O}$ PTICAL IMAGE sensors fail to provide visual information behind shelters, and they are easily discovered by hidden individuals. Real-time see-through-wall (STW) radars have therefore drawn increasing attention in homeland security, disaster rescue, and military industry-related applications to detect, locate, and monitor hidden individuals with electromagnetic waves [1]. A continuous-wave (CW) radar transmits an RF sinusoidal signal toward a target. The radar then receives and demodulates the echo signal to evaluate the relative velocity of the

\footnotetext{
Manuscript received July 09, 2012; revised October 29, 2012; accepted November 05, 2012. Date of publication December 19, 2012; date of current version January 17, 2013. This work was supported in part by the National Science Council, Taiwan, under Grant 100-2221-E-110-081-MY3, Grant 100-2221-E-110-082-MY3, and Grant 101-2622-E-110-005-CC3. This paper is an expanded paper from the IEEE MTT-S International Microwave Symposium, Montreal, QC, Canada, June 17-22, 2012.

F.-K. Wang and T.-S. Horng are with the Department of Electrical Engineering, National Sun Yat-Sen University, Kaohsiung 804, Taiwan (e-mail d983010020@student.nsysu.edu.tw; jason@ee.nsysu.edu.tw).

K.-C. Peng is with the Department of Computer and Communication Engineering, National Kaohsiung First University of Science and Technology, Kaohsiung 811, Taiwan (e-mail: peterpkg@ccms.nkfust.edu.tw).

J.-K. Jau, J.-Y. Li, and C.-C. Chen are with the Information and Communication Research Laboratories, Industrial Technology and Research Institute (ITRI), Hsinchu 310, Taiwan (e-mail: jekuan@itri.org.tw).

Color versions of one or more of the figures in this paper are available online at http://ieeexplore.ieee.org.

Digital Object Identifier 10.1109/TMTT.2012.2228223
}

target. Since the early 1970 s, CW radars have been widely used to noncontact monitor cardiopulmonary activity and search for alive victims under earthquake rubble. However, several technical difficulties, such as null detection points and co-frequency interference, are encountered in practical applications in which the robustness issue must be addressed. To overcome these difficulties, many architectures and demodulation methods have been developed at the cost of system complexity and power consumption [2]. Moreover, multiple targets in the environment are detected using the multiple antenna $\mathrm{CW}$ radar with advanced digital signal processing [3]-[5]. However, a tradeoff between robustness and efficiency is always incurred in operation. Actually, the $\mathrm{CW}$ radar is limited mainly by its inability to obtain range information for the target [6].

The above limitation of $\mathrm{CW}$ radars can be overcome by pulse radars or frequency-modulated continuous-wave (FMCW) radars. Pulse radars transmit a sequence of short pulses of RF energy, and estimate range to the target by measuring the time delay in returned pulses. Furthermore, pulse radars can simultaneously detect vital signs and exhibit high immunity to interference signals using the pulse generator with programmable delay and synchronization control [7]. However, these mechanisms significantly increase hardware complexity and the cost [8]. FMCW radars modulate the $\mathrm{CW}$ carrier frequency with a triangular waveform. Range information of the targets can then be acquired using the frequency difference between the transmitted and echo signals. However, the range correlation of phase noise for cancellation purpose is less satisfactory with the larger frequency difference between the transmitted and echo signals [9]. Therefore, FMCW generator usually requires an integer- $N$ phase-locked loop (PLL) referencing a direct digital frequency synthesizer (DFS) [10]-[12] or a fractional- $N$ PLL with a delta-sigma modulator [13] to achieve low phase noise, fast settling time, and precise frequency control. The generator is thus complex, and the costs and power consumption are high. Moreover, the clutter signals make it difficult to distinguish the key target from various objects within the sensing range. The clutter effect is often cancelled by generating a signal with the same amplitude but opposite phase as the clutter signal [14].

Approaches attempting to detect the azimuth of targets, including sequential lobing [15], conical scanning [16], and monopulse [17], compare the echo signals measured at different beam directions of a phased array antenna. The other approach, 


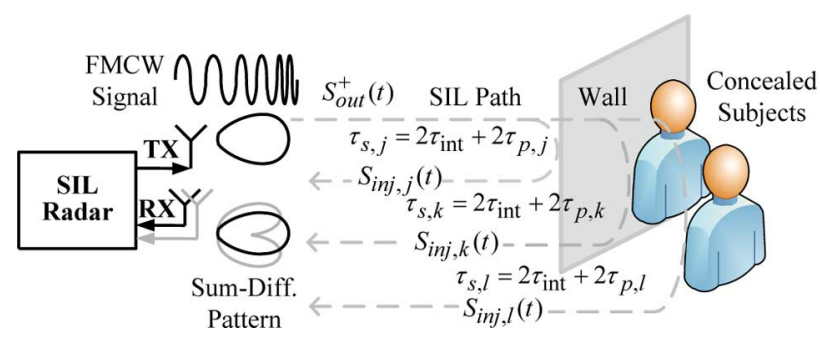

Fig. 1. Proposed STW imaging system using a SIL radar with FMCW and sum-difference pattern detection techniques.

synthetic aperture radar (SAR) [18], utilizes relative motion between the radar and the target. The echo signals captured successively at the different antenna positions are coherently detected and then post-processed to obtain an image of the target region. However, these approaches [15]-[18] normally use a large antenna array for finer spatial resolution. Therefore, the system complexity is also increased, leading to excessively high costs and power consumption.

Since the early 1990s, FMCW technology has been adopted in imaging radars to provide a real-time image with obscured line of sight [19]-[21]. Recent works [21] located a moving subject behind a wall by subtracting real-time output spectra to remove the clutter signals produced by stationary objects. However, an FMCW radar has difficulty in extracting tiny body movements, such as due to cardiopulmonary activity, from continuous range information, since the noise sideband of a transmitter often swamps the target Doppler signals [22]. On the other hand, some FMCW approaches detect the vital sign signals by demodulating the low-frequency information from the FM carrier frequency [23], [24], but fail to provide the synchronous range information.

In our previous works [25], [26], a new kind of CW radar has been introduced to detect vital signs. In its basic operation, an oscillator transmits a CW signal, which is partly reflected by a distant target, and then injected into the same oscillator to form a self-injection-locked (SIL) state. Doppler information of the target motion can be simply extracted from frequency demodulation of the oscillator output. This radar, called a SIL radar, was validated to achieve a high sensitivity with low system complexity. Additionally, our recent work [27] developed a SIL FMCW radar with simple hardware to detect a single concealed individual by using background subtraction of FMCW spectra measured in different time intervals. In this work, for the first time the FMCW and sum-difference pattern detection techniques are combined to construct a wall-transparent image to position multiple concealed individuals behind a shelter, as shown in Fig. 1. Since the SIL radar can detect Doppler shifts introduced by the cardiopulmonary activity of a human subject with a high signal-to-noise ratio (SNR) gain [25], the proposed system can therefore monitor the vital signs of concealed individuals simultaneously.

\section{SIL RADAR With RANGing AND TRACKING CAPABILITIES}

Fig. 2 shows block and timing diagrams of the proposed SIL radar, which contains a differential voltage-controlled oscillator (VCO) with an injection input, a low-noise amplifier (LNA)

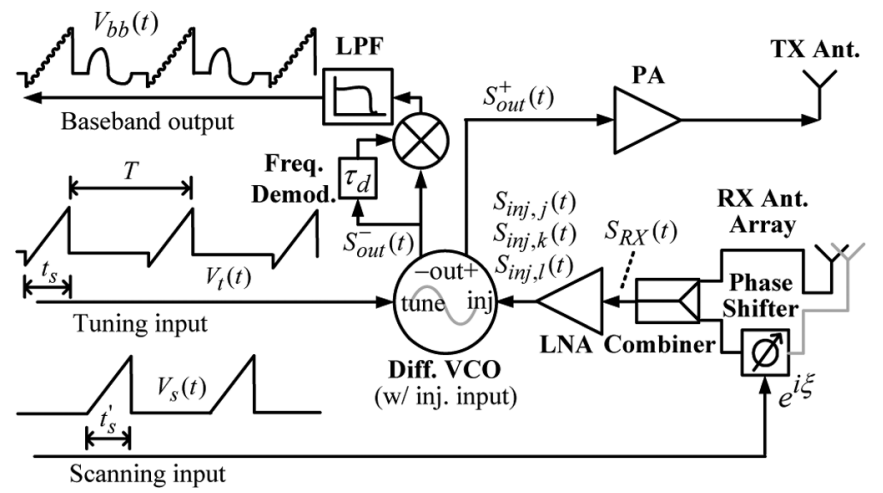

Fig. 2. Block and timing diagrams of the proposed SIL radar.

with a power gain of $14 \mathrm{~dB}$, a transmit (TX) antenna, a power amplifier (PA) with a power gain of $8 \mathrm{~dB}$, a receive $(\mathrm{RX})$ antenna array consisting of two elements, a phase shifter, a combiner to synthesize sum-difference pattern for azimuth detection, and a frequency demodulator composed of a mixer, delay element, and low-pass filter (LPF). Both the TX and RX antenna elements are horn antennas with a high gain of more than $10 \mathrm{dBi}$ from 2 to $18 \mathrm{GHz}$. The tuning range of the $\mathrm{VCO}$ ranges from 2.3 to $2.8 \mathrm{GHz}$, and it delivers an output power of approximately $0 \mathrm{dBm}$. The operating procedure of the proposed radar is described briefly as follows.

First, in the FMCW mode, the scanning input voltage $V_{s}(t)$ remains constant to fix the beam direction of the RX antenna array. In contrast, the tuning input voltage $V_{t}(t)$ outputs a ramp signal to frequency modulate the inherent oscillation signal $S_{\text {osc }}(t)$ of the VCO during the duration $t_{s}$. The positive output signal $S_{\text {out }}^{+}(t)$ of the VCO is amplified by the PA and then emitted by the TX antenna toward the subjects concealed behind a wall. According to Fig. 1, the signals $S_{\mathrm{RX}}(t)$ reflected from the wall and the subjects are received by the RX antenna array and then amplified as the injection signals, $S_{\mathrm{inj}, j}(t)$, $S_{\mathrm{inj}, k}(t)$, and $S_{\mathrm{inj}, l}(t)$, respectively, by the LNA. Next, these injection signals enter the $\mathrm{VCO}$ via its injection input and cause the VCO to enter a SIL state. Round trip propagation delay of the SIL path with respect to $S_{\mathrm{inj}, j}(t), S_{\mathrm{inj}, k}(t)$, and $S_{\mathrm{inj}, l}(t)$ is $\tau_{s, j}, \tau_{s, k}$, and $\tau_{s, l}$, respectively. Notably, the SIL state fails to hold once the injection signal frequency exceeds the locking range, which may occur for a very short sweeping duration $t_{s}$.

Second, the radar is switched to CW mode by setting $V_{t}(t)$ as constant voltage $V_{t}^{\prime}$. A ramp signal of $V_{s}(t)$ enters the voltagecontrolled phase shifter for detecting Doppler shifts with different antenna beam directions during the duration $t_{s}^{\prime}$. Furthermore, the echo signals inject into the VCO to make the VCO achieve a SIL state.

Third, the negative output signal $S_{\text {out }}^{-}(t)$ of the VCO is fed into the frequency demodulator to output a baseband signal $V_{b b}(t)$ that reflects the frequency variation of the $\mathrm{VCO}$ in the FMCW and $\mathrm{CW}$ modes of the radar. After signal processing, the radar finally extracts the range information of the subjects by using background subtraction of the FMCW spectra measured one time per $T$ seconds. Furthermore, the radar uses the sum-difference pattern detection technique to track the azimuth of the individual subjects in the $\mathrm{CW}$ mode. 


\section{A. Range Detection in the FMCW Mode}

In Fig. 2, the VCO has an inherent instantaneous oscillation frequency $\omega_{\text {osc }}(t)$ and a constant oscillation amplitude $V_{\text {osc }}$. When an injection signal $S_{\mathrm{inj}}(t)$, with instantaneous frequency $\omega_{\text {inj }}(t)$ and constant amplitude $V_{\text {inj }}$, enters the VCO, output signal $S_{\text {out }}(t)$, with constant amplitude $V_{\text {out }}$, can be regarded as a vector that rotates clockwise with beat frequency $d \alpha(t) / d t$ with respect to $S_{\mathrm{inj}}(t)$, while $\alpha(t)$ is the phase difference from $S_{\text {inj }}(t)$ to $S_{\text {osc }}(t)$. Based on our previous work [27], the instantaneous output frequency $\omega_{\text {out }}(t)$ is represented as

$$
\omega_{\text {out }}(t)=\omega_{\text {osc }}(t)-\omega_{\mathrm{LR}}(t) \sin \alpha(t) .
$$

In (1), the locking range $\omega_{\mathrm{LR}}(t)$ for a $\mathrm{VCO}$ is given by

$$
\omega_{\mathrm{LR}}(t)=\frac{\omega_{\mathrm{osc}}(t)}{2 Q \operatorname{tank}} \frac{V_{\mathrm{inj}}}{V_{\mathrm{osc}}}
$$

where $Q_{\text {tank }}$ denotes the quality factor of the VCO's tank circuit. The ramp signal of $V_{t}(t)$ is applied to the VCO's tuning terminal, and the radar is operated in the FMCW mode. The oscillation frequency of the VCO can be expressed as

$$
\omega_{\mathrm{osc}}(t)=\omega_{\mathrm{osc}}+2 \pi K_{v} V_{t}(t)
$$

where $K_{v}$ is the tuning sensitivity and $\omega_{\text {osc }}$ is the center frequency of VCO. Consider a situation in which the injection signal is a summation of multiple echo signals from various targets (Fig. 1). Thus, (1) can be rewritten as

$$
\omega_{\mathrm{out}}(t)=\omega_{\mathrm{osc}}(t)-\sum_{i} \omega_{\mathrm{LR}, i}(t) \sin \alpha_{i}(t)
$$

where

$$
\omega_{\mathrm{LR}, i}(t)=\frac{\omega_{\mathrm{osc}}(t)}{2 Q_{\mathrm{tank}}} \frac{V_{\mathrm{inj}, i}}{V_{\mathrm{osc}}} .
$$

The phase difference $\alpha_{i}(t)$ under a SIL condition is equal to the propagation phase delay of each injection signal $S_{\mathrm{inj}, i}(t)$, and can be expressed as

$$
\alpha_{i}(t)=\left\{\begin{array}{l}
\omega_{\mathrm{osc}}(t)\left(2 \tau_{\mathrm{int}}+2 \tau_{p, i}\right), \quad \text { for } i=j \\
\omega_{\mathrm{osc}}(t)\left(2 \tau_{\mathrm{int}}+2 \tau_{p, i}+2 \Delta \tau_{p, i}(t)\right),
\end{array} \text { for } i=k, l\right.
$$

where $2 \tau_{\text {int }}$ and $2 \tau_{p, i}$ are the round-trip delay within the internal circuit and the $i$ th SIL path delay, respectively. Additionally, $\Delta 2 \tau_{p, i}(t)$ for $i=k, l$ represents the delay fluctuation caused by body motion of the subjects. Under a small-angle approximation for $\omega_{\text {osc }}(t) \Delta \tau_{p, i}(t)$, the frequency-demodulated and low-pass filtered baseband output signal, $V_{b b}(t)$, is proportional to the instantaneous oscillation frequency variation and can be found as

$$
V_{b b}(t)=G_{c} V_{\text {out }}\left(\omega_{\text {out }}(t)-\omega_{\text {osc }}\right) \tau_{d} \approx G_{r} V_{t}(t)+V_{m}(t)
$$

where $G_{r}=2 \pi G_{c} K_{v} V_{\text {out }} \tau_{d}$, and $G_{c}$ and $\tau_{d}$ represent the conversion loss of the mixer and the time delay offered by the delay element, respectively, in the frequency demodulator. For
$2 \omega_{\text {osc }}(t) \Delta \tau_{p, i}(t) \ll 1$, the demodulated signal in (7) can be approximated by

$$
V_{m}(t) \approx \sum_{i} V_{m, i}(t)+\sum_{i=k, l} \Delta V_{m, i}(t)
$$

where

$$
V_{m, i}(t)=-G_{c} V_{\text {out }} \tau_{d} \omega_{\mathrm{LR}, i}(t) \sin \left(\omega_{\mathrm{osc}}(t)\left(2 \tau_{\mathrm{int}}+2 \tau_{p, i}\right)\right)
$$

and

$$
\begin{aligned}
\Delta V_{m, i}(t)=-G_{c} V_{\mathrm{out}} \tau_{d} \omega_{\mathrm{LR}, i}(t) & \cos \left(\omega_{\mathrm{osc}}(t)\left(2 \tau_{\mathrm{int}}+2 \tau_{p, i}\right)\right) \\
& \times \sin \left(2 \omega_{\mathrm{osc}}(t) \Delta \tau_{p, i}(t)\right)
\end{aligned}
$$

Since one sweeping duration $t_{s}$ is significantly shorter than the period of vital signs, $2 \Delta \tau_{p, k}(t)$ and $2 \Delta \tau_{p, l}(t)$ can be treated as a constant with time within the duration $t_{s}$. Hence, $\Delta V_{m, k}(t)$ and $\Delta V_{m, l}(t)$ can be mathematically merged into $V_{m, k}(t)$ and $V_{m, l}(t)$, respectively, in discrete time. Consequently, $V_{m}(t)$ in (7) can be extracted after subtracting $G_{r} V_{t}(t)$ from $V_{b b}(t)$ in the baseband. By assuming that the VCO has a small tuning range, the locking range, $\omega_{\mathrm{LR}, i}(t) \approx \omega_{\mathrm{LR}, i}=\omega_{\mathrm{osc}} V_{\mathrm{inj}, i} /\left(2 Q_{\mathrm{tank}} V_{\mathrm{osc}}\right)$, can be approximated. Moreover, the Fourier transform of $V_{m}(t)$ is

$$
\begin{aligned}
V_{m}(\omega)= & \mathbb{F}\left(w(t) V_{m}(t)\right) \\
= & -G_{c} V_{\mathrm{out}} \tau_{d} \sum_{i} \frac{\omega_{\mathrm{LR}, i}}{2 j} \\
& \times\left(W\left(\omega-\omega_{s, i}\right) e^{j \omega_{\mathrm{osc}} \tau_{s, i}}-W\left(\omega+\omega_{s, i}\right) e^{-j \omega_{\mathrm{osc}} \tau_{s, i}}\right)
\end{aligned}
$$

where $w(t)$ and $W(\omega)$ represent the Fourier transform pair of a window function, and $\omega_{s, i}$, each tone's center frequency of the detection spectrum, equals $2 \pi S_{R} \tau_{s, i}$, where $S_{R}$ is the scan rate (i.e., rate of change of $K_{v} V_{t}(t)$ per $t_{s}$ ). Notably, $\omega_{s, i}$ is related to the $i$ th SIL path delay. Therefore, the distance detected for the targets within the sensing range can be calculated and expressed as

$$
R_{p, i}=c \tau_{p, i}=c\left(\frac{\omega_{s, i}}{4 \pi S_{R}}-\tau_{\mathrm{int}}\right)
$$

where $c$ denotes the speed of light.

Next, the proposed theory is verified based on the baseband output signal measurements. In contrast to Fig. 2, the TX antenna and RX antenna array are bypassed. The experimental setup connects the PA output directly to the LNA input through a variable time delay element and an attenuator to adjust the SIL path delay as well as the injection amplitude. In the experiment, the VCO with a tank quality factor of about 24 sweeps from 2.4 to $2.7 \mathrm{GHz}$ in $0.01 \mathrm{~s}$; the scan rate is thus $30 \mathrm{MHz} / \mathrm{ms}$. The SIL path delay and the injection-to-oscillation amplitude ratio $V_{\mathrm{inj}, i} / V_{\mathrm{osc}}$ is set as $28.4 \mathrm{~ns}$ and 0.1 , respectively. Therefore, the calculated locking range $\omega_{\mathrm{LR}, i}$ is around $2 \pi \times 5 \mathrm{MHz}$ to $2 \pi \times 5.625 \mathrm{MHz}$ due to the variation in $\omega_{\text {osc }}(t)$. In Fig. 3 , the black line expresses the calculated baseband output waveform 


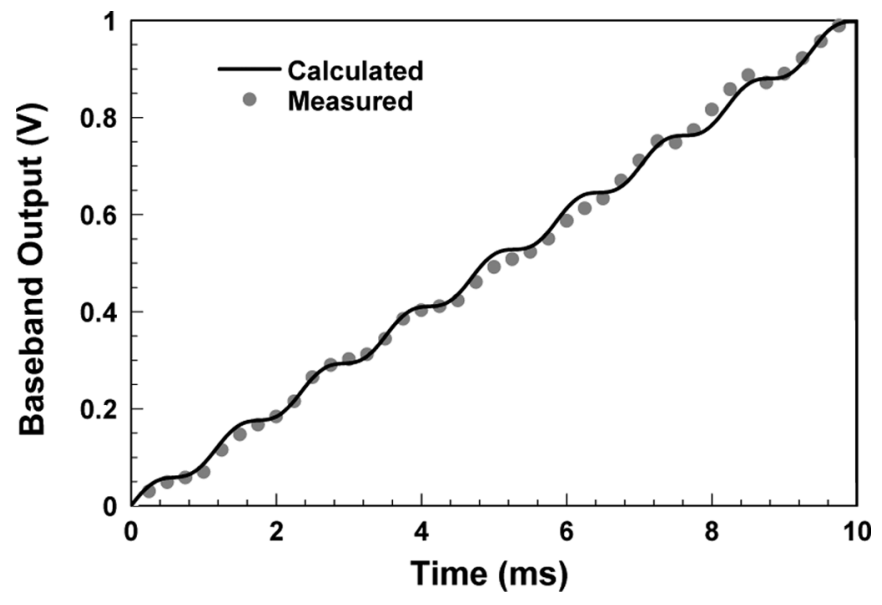

Fig. 3. Baseband output waveform of SIL radar in the FMCW mode.

according to (7) with the aforementioned parameters. The amplitude of ripple $V_{m}(t)$ is proportional to the locking range. Additionally, the extracted FMCW spectrum shows a tone centered at $852 \mathrm{~Hz}$. The measured results denoted by the circle symbols agree well with the calculated waveform.

\section{B. Azimuth Detection in the CW Mode}

Assume that $V_{t}(t)$ is fixed as a constant value, $V_{t}^{\prime}$. The SIL radar is thus operated in the $\mathrm{CW}$ mode with the following oscillation frequency and locking range with respect to the $\mathrm{VCO}$

$$
\omega_{\mathrm{osc}}^{\prime}=\omega_{\mathrm{osc}}+2 \pi K_{v} V_{t}^{\prime}, \quad \omega_{\mathrm{LR}, i}^{\prime}=\frac{\omega_{\mathrm{osc}}^{\prime} V_{\mathrm{inj}, i}}{\left(2 Q_{\mathrm{tank}} V_{\mathrm{osc}}\right)} .
$$

According to (7) and (8), $V_{b b}(t)$ can be rewritten as

$$
V_{b b}(t) \approx V_{\mathrm{dc}}+\sum_{i=k, l} \Delta V_{m, i}(t)
$$

where

$$
\begin{aligned}
V_{\mathrm{dc}}=G_{c} & V_{\mathrm{out}} \tau_{d} \\
& \times\left[2 \pi K_{v} V_{t}^{\prime}-\sum_{i} \omega_{\mathrm{LR}, i}^{\prime} \sin \left(\omega_{\mathrm{osc}}^{\prime}\left(2 \tau_{\mathrm{int}}+2 \tau_{p, i}\right)\right)\right]
\end{aligned}
$$

and

$$
\begin{array}{r}
\Delta V_{m, i}(t)=-G_{c} V_{\mathrm{out}} \tau_{d} \omega_{\mathrm{LR}, i}^{\prime} \cos \left(\omega_{\mathrm{osc}}^{\prime}\left(2 \tau_{\mathrm{int}}+2 \tau_{p, i}\right)\right) \\
\times \sin \left(2 \omega_{\mathrm{osc}}^{\prime} \Delta \tau_{p, i}(t)\right) .
\end{array}
$$

In (16), $\Delta \tau_{p, i}(t)$ can be expressed as

$$
\Delta \tau_{p, i}(t)=\frac{\Delta R_{p, i}(t)}{c}
$$

where $\Delta R_{p, i}(t)$ denotes position fluctuation of the subject with respect to the original distance. The dc level $V_{\mathrm{dc}}$ in (14) is the so-called dc offset, which greatly degrades the system performance. Clearly, according to (15), $V_{t}^{\prime}$ can be particularly tuned for eliminating the dc offset.

As shown in Fig. 4, the proposed radar utilizes one TX and two RX antennas for azimuth detection in the $\mathrm{CW}$ mode. All

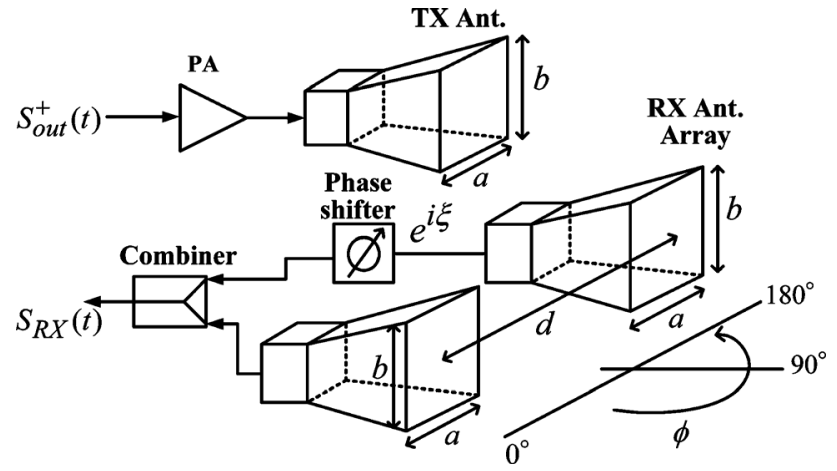

Fig. 4. TX and RX antennas in the proposed SIL radar.

of the elements are horn antennas with aperture width $a$ and height $b . S_{\text {out }}^{+}(t)$ is power amplified, emitted by the TX antenna, reflected from the targets in the environment, and received separately by the two identical RX antenna elements spaced at distance $d$ apart. Assume that the phase shifter is ideally lossless; hence, the transmission coefficient is $e^{i \xi}$, and $\xi$ is the voltage-controlled phase shift. The two received signals are then combined as $S_{\mathrm{RX}}(t)$ for injecting into the VCO. Assume that the zenith angle remains constant. The RX antenna array pattern $E(\phi, \xi)$ can thus be written as

$$
E(\phi, \xi)=F(\phi) A(\phi, \xi)
$$

where the element factor $F(\phi)$, horn antenna pattern function [28] in this case, is given by

$$
F(\phi)=\frac{\pi^{2}}{2}(1+\sin \phi) \frac{\cos \left(\frac{a \cdot \omega_{\mathrm{osc}}^{\prime}}{2 c} \cos \phi\right)}{\pi^{2}-4\left(\frac{a \cdot \omega_{\mathrm{osc}}^{\prime}}{2 c} \cos \phi\right)^{2}}
$$

and the array factor $A(\phi, \xi)$ in (18) is

$$
A(\phi, \xi)=\cos \left[\frac{1}{2}\left(\frac{\omega_{\mathrm{osc}}^{\prime} d}{c} \cos \phi+\xi\right)\right] .
$$

Notably, the azimuth $\phi$ in (19) ranges from $0^{\circ}$ to $180^{\circ}$. In the experiment, the horn antennas with $a$ of $10 \mathrm{~cm}$ and $b$ of 7.5 $\mathrm{cm}$ are used as antenna elements, where $\omega_{\text {osc }}^{\prime}$ is chosen around $2 \pi \times 2.4 \mathrm{GHz}$; in addition, the spacing between two RX antenna elements is about $10.6 \mathrm{~cm}$. Fig. 5 compares the radiation pattern with $\xi$ equal to $0^{\circ}, 90^{\circ}, 180^{\circ}$, and $270^{\circ}$, respectively, in which the measurement results and theoretical predictions correlate well with each other.

Next, the tracking pattern $P\left(\phi_{0}, \xi\right)$ for a target located at an azimuth of $\phi_{0}$ is defined as the ratio of sum to difference pattern by varying the phase shift $\xi$ at the azimuth of $\phi=\phi_{0}$. Thus, this pattern is given as

$$
\begin{aligned}
P\left(\phi_{0}, \xi\right) & =\left|\frac{E\left(\phi_{0}, \xi\right)}{E\left(\phi_{0}, \xi+180^{\circ}\right)}\right| \\
& =\left|\cot \left[\frac{1}{2}\left(\frac{\omega_{\mathrm{osc}}^{\prime} d}{c} \cos \phi_{0}+\xi\right)\right]\right|
\end{aligned}
$$




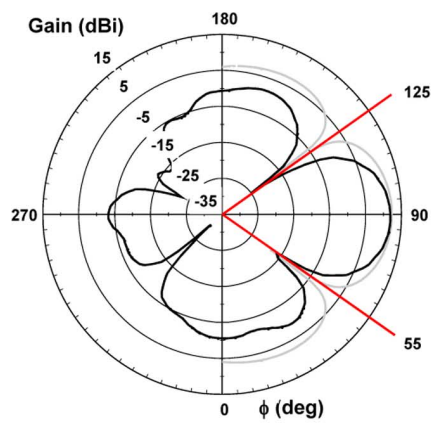

(a)

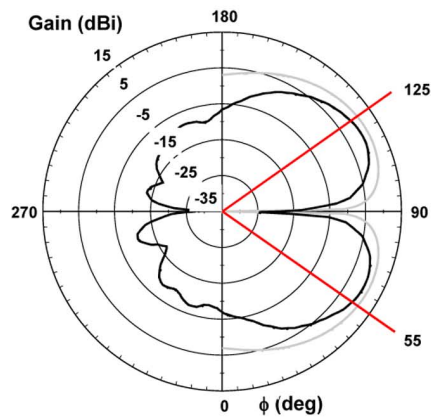

(c)

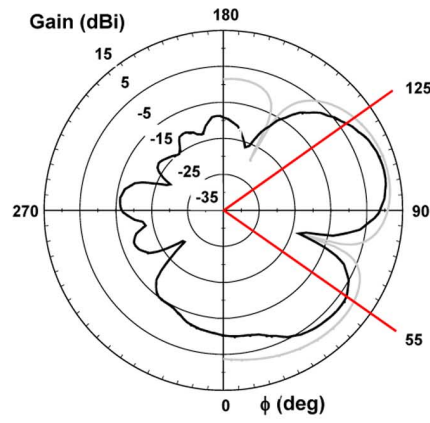

(b)

(d)

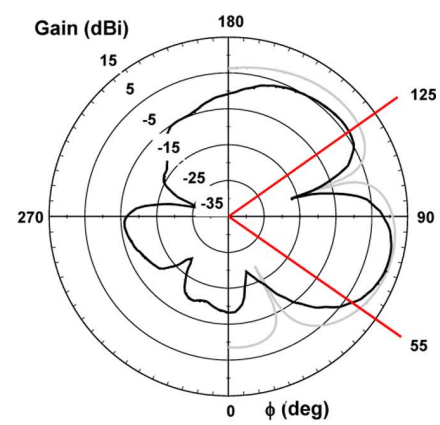

Fig. 5. Scanned radiation patterns of the two-element array for the reception (measurement: black - , theory: gray -). (a) $\xi=0^{\circ}$. (b) $\xi=90^{\circ}$. (c) $\xi=$ $180^{\circ}$. (d) $\xi=270^{\circ}$.

Moreover, the tracking azimuth angle $\phi_{t}$, in which the tracking pattern shows an infinite peak amplitude, is found by

$$
\frac{\omega_{\mathrm{osc}}^{\prime} d}{c} \cos \phi_{t}+\xi=0 .
$$

Substituting (22) into (21) yields

$$
P\left(\phi_{0}, \phi_{t}\right)=\left|\cot \left[\frac{\omega_{\mathrm{osc}}^{\prime} d}{2 c}\left(\cos \phi_{0}-\cos \phi_{t}\right)\right]\right|
$$

and the output baseband amplitude is proportional to $P\left(\phi_{0}, \phi_{t}\right)$. In the experiment, $\omega_{\text {osc }}^{\prime} d / c$ is around $1.7 \pi$. Hence, the position of sum pattern scans from $53.97^{\circ}$ to $126.03^{\circ}$ with $\xi$ varying from $-180^{\circ}$ to $180^{\circ}$. Similarly, the difference pattern has the same scanning range when $\xi$ varies from $0^{\circ}$ to $360^{\circ}$. To avoid the judgment errors, this work selects $\phi_{t}$ range as $55^{\circ}$ to $125^{\circ}$. We recommend that future works expand the tracking azimuth by designing a miniaturized high-gain antenna element to further reduce the spacing between the two RX antenna elements.

\section{EXPERIMENTAL RESULTS}

Figs. 6 and 7 show the actual photograph and the top view sketch of the experimental setup. The SIL radar in the FMCW mode sweeps the VCO oscillation frequency from 2.4 to $2.7 \mathrm{GHz}$ with $30-\mathrm{MHz} / \mathrm{ms}$ scan rate; in addition, $\xi$ is fixed as $0^{\circ}$ to maintain the beam direction $\phi$ equal to $90^{\circ}$. The internal circuit delay, $2 \tau_{\text {int }}$, is approximately $15.5 \mathrm{~ns}$. During the experiment, all of the targets are sheltered by a $180 \times 200 \times 3 \mathrm{~cm}^{3}$ wooden partition wall. Within the sensing range, a subject is seated still and breathing normally, with various objects causing clutter signals and a wood wall having

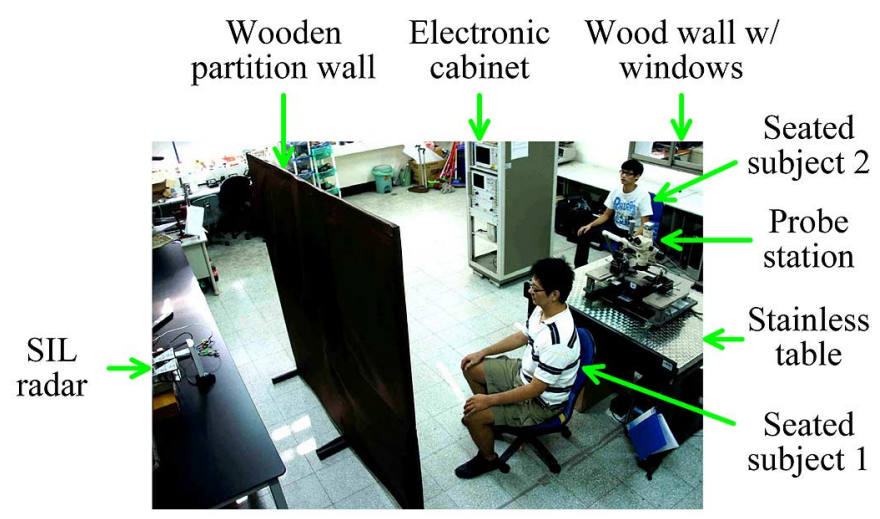

Fig. 6. Actual photograph of the experimental setup for detection of concealed individuals.

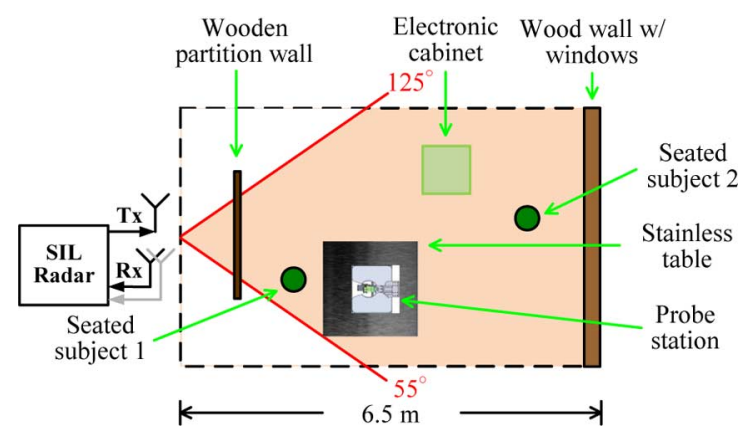

Fig. 7. Top view sketch of Fig. 6.

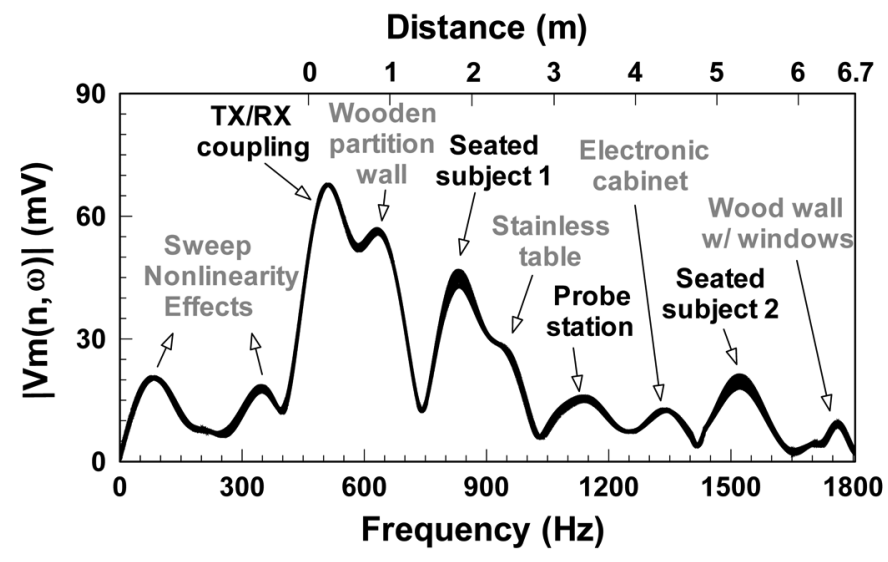

Fig. 8. Dynamic FMCW spectra for range detection in the scenario of Fig. 6.

windows located at the far edge. Distances from the radar to the wooden partition wall, seated subject 1 , stainless table, probe station, electronic cabinet, seated subject 2, and wood wall with windows are $0.83,1.86,2.41,3.4,4.41,5.28$, and $6.5 \mathrm{~m}$, respectively. Additionally, the azimuth angle of seated subjects 1 and 2 are located at $65^{\circ}$ and $95^{\circ}$, respectively.

In the FMCW mode, the sweeping duration $t_{s}$ and the spectrum sampling period $T$ are set as 0.01 and $0.1 \mathrm{~s}$, respectively. Fig. 8 shows the 1 -s cumulative FMCW spectra. The tones located at $630,836,945,1140,1345,1521$, and $1760 \mathrm{~Hz}$ are derived from the TX/RX coupling signal and the echo signals of the wooden partition wall, seated subject 1 , stainless table, probe station, electronic cabinet, seated subject 2 , and wood wall with 


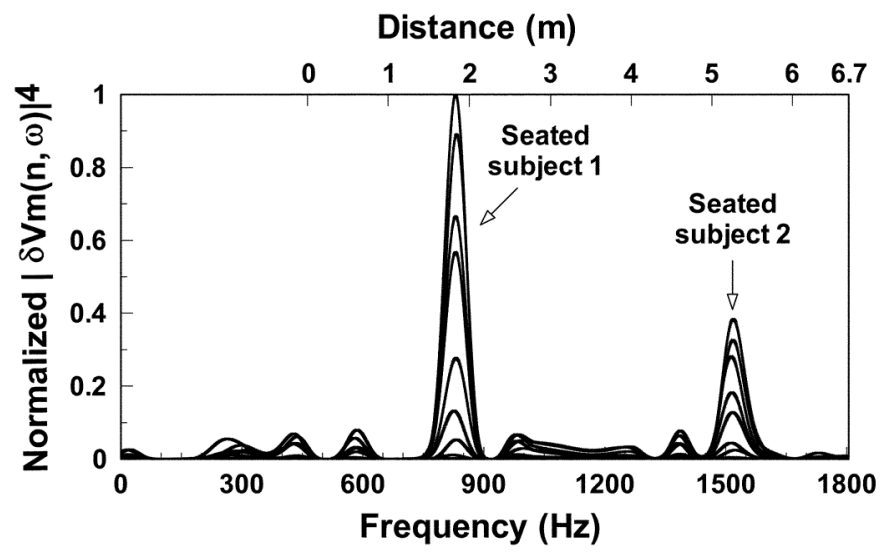

Fig. 9. Fourth power of the background subtracted spectra with respect to the original spectrum in Fig. 8.

windows, respectively. According to (12), their detected distances are $0.825,1.855,2.4,3.375,4.4,5.28$, and $6.475 \mathrm{~m}$, respectively. The maximum range error is less than $3 \mathrm{~cm}$. Moreover, the range resolution can be further enhanced by using a broader scanning bandwidth. In particular, the tones located below $465 \mathrm{~Hz}$ originate mainly from the sweep nonlinearity of the VCO. To remove the effects of sweep nonlinearity and stationary clutter, this work defines background subtracted spectrum as

$$
\begin{gathered}
\left|\delta V_{m}(n, \omega)\right|=\left|\mathbb{F}\left(w(t-n T) V_{m}(t)\right)-\mathbb{F}\left(w(t) V_{m}(t)\right)\right|, \\
n \in N .
\end{gathered}
$$

With an emphasis on the spectral peak variations in relation to spectral subtraction noise, Fig. 9 shows the normalized fourth power of background subtracted spectra with respect to Fig. 8 . Clearly, the largest two peaks of spectral variations occur in the frequencies that represent the distances of the seated subjects 1 and 2 from the radar, owing to their cardiopulmonary signals during the 1-second measurement time. Notably, in such a short measurement time, the maximum peaks corresponding to the maximum cardiopulmonary displacements appeared in the two seated subjects may not occur at the same plot. Moreover, in Fig. 9, the spectral peak amplitude shown for the seated subject 1 varies more severely than for the seated subject 2 because the former is closer in distance to the SIL radar. Figs. 10(a) and 11(a) record the position fluctuations with the transformed spectra shown in Figs. 10(b) and 11(b) for the seated subjects 1 and 2, respectively. The spectral peak values associated with respiration and heartbeat are 7.132 and $1.324 \mathrm{~mm}$, respectively, for the seated subject 1 , and 8.47 and $1.766 \mathrm{~mm}$, respectively, for the seated subject 2. Additionally, Figs. 10(b) and 11(b) clearly indicates a breathing rate of 18 breaths/min and a heartbeat rate of 76 beats/min for the seated subject 1 , and a breathing rate of 17 breaths $/ \mathrm{min}$ and a heartbeat rate of 72 beats $/ \mathrm{min}$ for the seated subject 2 , respectively.

Next, the SIL radar is switched to the CW mode by setting $V_{t}(t)=V_{t}^{\prime}$ and $\omega_{\mathrm{osc}}(t)=\omega_{\mathrm{osc}}^{\prime}$. The tracking azimuth $\phi(t)$ is then scanned from $55^{\circ}$ to $125^{\circ}$ by using $V_{s}(t)$ to vary the phase shift $\xi$. Fig. 12 shows the measured ratio of sum to difference pattern voltages with varying tracking azimuth. According to

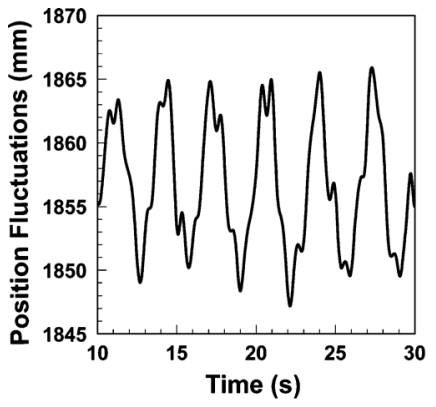

(a)

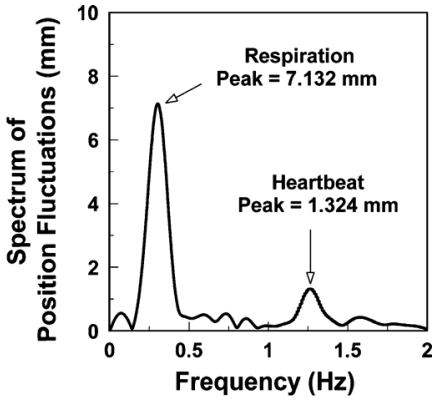

(b)
Fig. 10. Vital-sign detection results for the seated subject 1 in Fig. 6. (a) Subject's position fluctuations due to vital signs. (b) Spectrum of position fluctuations.

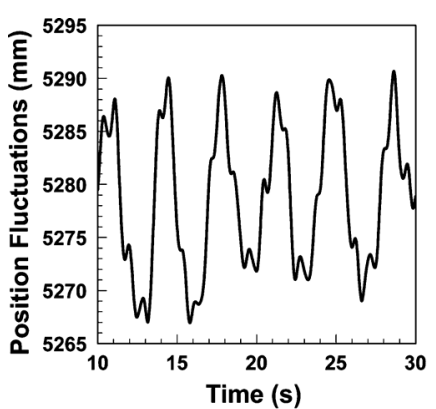

(a)

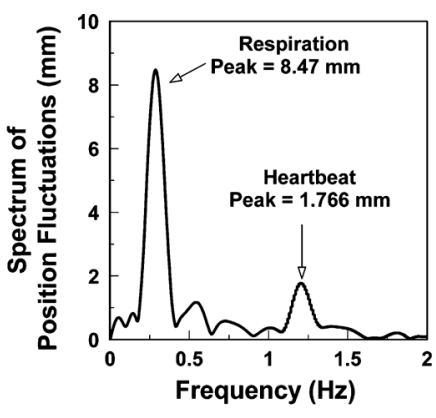

(b)
Fig. 11. Vital-sign detection results for the seated subject 2 in Fig. 6. (a) Subject's position fluctuations due to vital signs. (b) Spectrum of position fluctuations.

Fig. 5, the width of main antenna beam is so wide that the received signal for various $\phi_{t}$ simultaneously contains the Doppler shifts caused by the two seated subjects located at different azimuth angles of $65^{\circ}$ and $95^{\circ}$, respectively. Since the tracking pattern is proportional to the measured voltage caused by the subjects' cardiopulmonary signals within the sensing range, Fig. 12 is dominated by the closer subject 1 at an azimuth of $65^{\circ}$, making it difficult to distinguish the other subject at an azimuth of $95^{\circ}$.

The STW image for positioning the seated subjects can be constructed based on Figs. 9 and 12 that show the range and azimuth information, respectively, by the following equation in polar form:

$$
I_{\mathrm{STW}}\left(R, \phi_{t}\right)=\left|\delta V_{m}(n, \omega(R))\right|^{4} \cdot P\left(\phi_{0}, \phi_{t}\right)
$$

where $\omega(R)$ is derived from (12) as $\omega=4 \pi S_{R}\left(R / c+\tau_{\text {int }}\right)$. The purpose of using the fourth power of the subtracted spectrum in (25) is to enhance the signal to clutter plus noise ratio of the constructed STW images. As dynamic FMCW spectra are measured per $0.1 \mathrm{~s}$, this prototype can provide $10-\mathrm{Hz}$ image rate to monitor the position information of concealed individuals in real-time. As a result, the STW image based on (25) provides colored blobs to position slow-moving or stationary subjects and suppresses the appearance of stationary objects. Fig. 13 shows the most distinctive STW image measured within 1-s monitoring period, in which the colored blobs should closely correspond to the position of the seated subjects in Fig. 7. However, in Fig. 13, the blob representative of the seated subject 1 


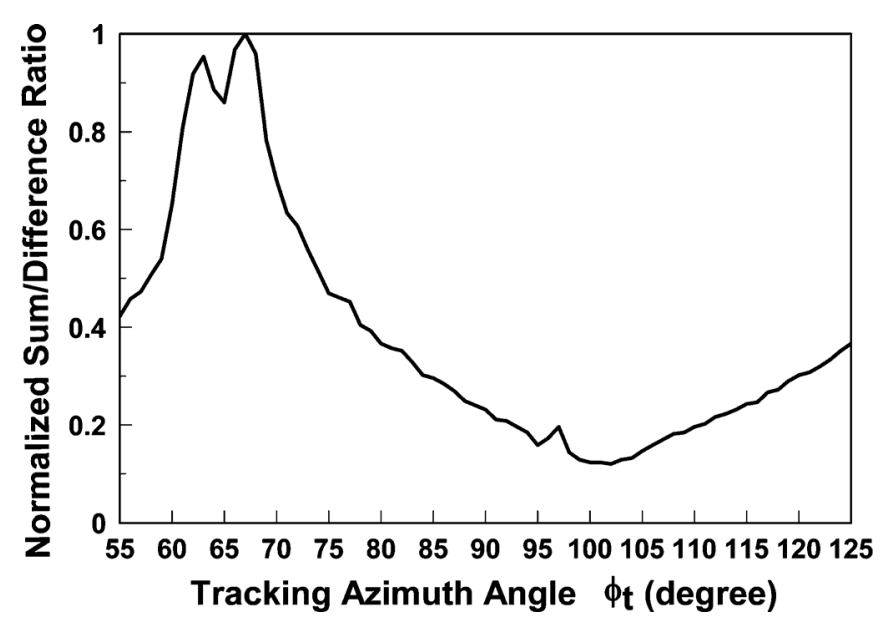

Fig. 12. Uncorrected tracking pattern for azimuth detection of the seated subjects in Fig. 6.

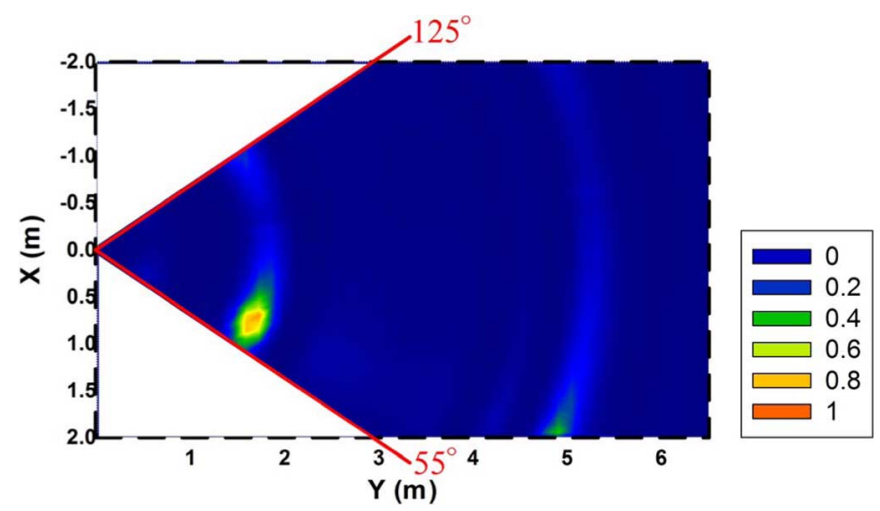

Fig. 13. Uncorrected STW image for position detection of the seated subjects in Fig. 6.

expands, owing to the significant distortion in the azimuth resolution, as shown in Fig. 12. Furthermore, the position of the seated subject 2 is mistaken as $5.28(\mathrm{~m}) \angle 65^{\circ}$ in polar form. In sum, the above approach fails in the two-person detection.

To resolve this problem, this work develops a time-domain correction scheme. Assume that the period of antenna beam scanning in the $\mathrm{CW}$ mode is significantly less than that of vital signals. The vital-sign signals detected in sum and difference pattern, $V_{\text {sum }}(\xi, t)$ and $V_{\text {diff }}(\xi, t)$, are given by

$$
V_{\text {sum }}(\xi, t)=E\left(\phi_{01}, \xi\right) V_{v s, 1}(t)+E\left(\phi_{02}, \xi\right) V_{v s, 2}(t)
$$

and

$$
\begin{aligned}
V_{\mathrm{diff}}(\xi, t)=E\left(\phi_{01}, \xi+\right. & \left.180^{\circ}\right) V_{v s, 1}(t) \\
& +E\left(\phi_{02}, \xi+180^{\circ}\right) V_{v s, 2}(t)
\end{aligned}
$$

where $E\left(\phi_{01}, \xi\right)$ and $E\left(\phi_{02}, \xi\right)$ denote the antenna factors in sum pattern for detecting cardiopulmonary signals of the seated subjects 1 and 2, respectively. While the array is operated in a difference pattern, the antenna factors are $E\left(\phi_{01}, \xi+180^{\circ}\right)$ and $E\left(\phi_{02}, \xi+180^{\circ}\right)$, respectively, for individual detections. Since $V_{v s, 1}(t)$ and $V_{v s, 2}(t)$ are obtained as known functions of vital signs from Figs. 10(a) and

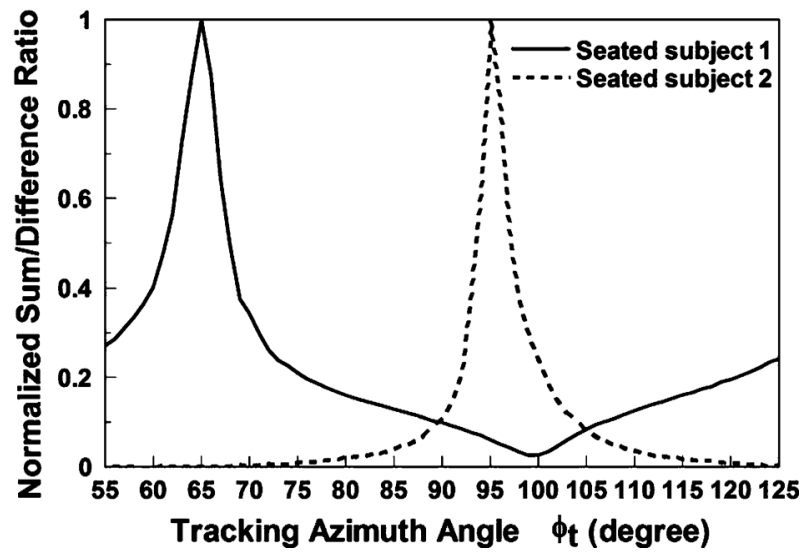

Fig. 14. Corrected tracking pattern for azimuth detection of the seated subjects in Fig. 6.

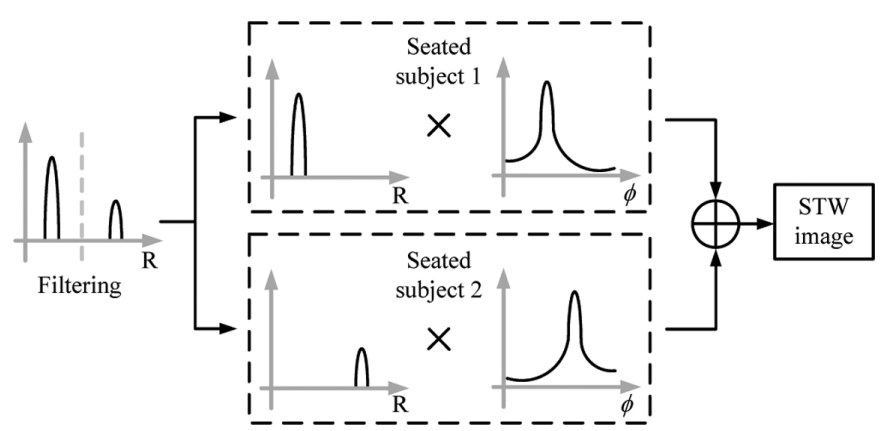

Fig. 15. STW imaging process for two different subjects.

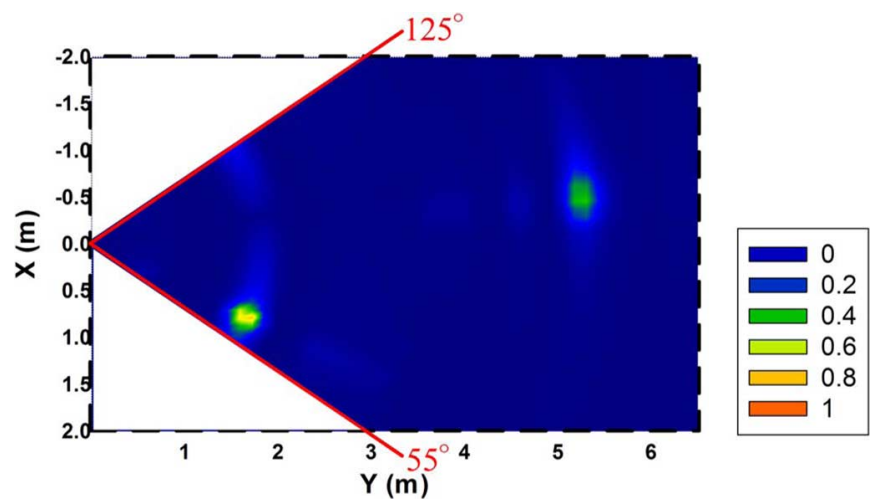

Fig. 16. Corrected STW image for position detection of the seated subjects in Fig. 6.

11(a), the antenna factors $E\left(\phi_{01}, \xi\right), E\left(\phi_{02}, \xi\right)$ for sum pattern and $E\left(\phi_{01}, \xi+180^{\circ}\right), E\left(\phi_{02}, \xi+180^{\circ}\right)$ for difference pattern can be derived from solving (26) and (27), respectively, at any two sampling times. Thereafter, the tracking patterns, $P\left(\phi_{01}, \phi_{t}\right)=E\left(\phi_{01}, \xi\right) / E\left(\phi_{01}, \xi+180^{\circ}\right)$ and $P\left(\phi_{02}, \phi_{t}\right)=E\left(\phi_{02}, \xi\right) / E\left(\phi_{02}, \xi+180^{\circ}\right)$, can be found as Fig. 14, showing a peak value at the azimuth of the seated subjects 1 and $2,65^{\circ}$ and $95^{\circ}$, respectively. This results agrees very well with the actual situation.

Fig. 15 shows the STW imaging process for two different subjects. First, $\left|\delta V_{m}(n, \omega)\right|^{4}$ is split by low- and high-pass filtering; in addition, both cutoff frequencies are set as $0.5\left(\omega_{s, k}+\omega_{s, l}\right)$ to isolate the range information of individual subjects. Second, 
these two split signals multiplied by the corresponding tracking pattern provide the positioning information. Finally, the two data sets from the subjects are summed to construct the STW image, as shown in Fig. 16. In this figure, positions of the two seated subjects are obvious, although the blob image of the seated subject 2 has a lower intensity due to a farther position from the radar, and hence, with a weaker echo signal. Moreover, the optical transfer function of the blob images in Fig. 16 can be improved by raising the spatial resolution with increased scanning bandwidth and antenna array elements. Notably, the brightness of the blob image varies with time according to the detected cardiopulmonary activity. Therefore, the two blob images twinkle asynchronously because the cardiopulmonary signals of two subjects have different periods.

\section{CONCLUSION}

This paper has presented a SIL radar with a one-element antenna for transmission and a two-element array antenna for reception to construct STW images in order to detect concealed individuals by their vital signs. The radar performs the range detection of different subjects by using background subtraction of FMCW spectra. Moreover, the radar uses the sum-difference pattern detection in the $\mathrm{CW}$ mode to monitor the azimuth angle of individual subjects. Despite the seriously distorted sum-difference pattern detection results for multiple subjects due to their mutual interference in the reception of multiple Doppler signals, this work has developed a time-domain correction scheme to overcome this problem. Importantly, the proposed radar imaging system uniquely positions multiple subjects and monitors their vital signs simultaneously, even when it is concealed from vision.

\section{REFERENCES}

[1] L.-P. Song, C. Yu, and Q. H. Liu, "Through-wall imaging (TWI) by radar: 2-D tomographic results and analyses," IEEE Trans. Geosci. Remote Sens., vol. 43, no. 12, pp. 2793-2798, Dec. 2005.

[2] C. Li, J. Cummings, J. Lam, E. Graves, and W. Wu, "Radar remote monitoring of vital signs," IEEE Microw. Mag., vol. 10, no. 1, pp. 47-56, Feb. 2009.

[3] O. Boric-Lubecke, V. M. Lubecke, A. Host-Madsen, D. Samardzija, and K. Cheung, "Doppler radar sensing of multiple subjects in single and multiple antenna systems," in 7th Int. Telecommun. Modern Satellite, Cable, Broadcast. Services Conf., Sep. 2005, pp. 7-11.

[4] Q. Zhou, J. Liu, A. Host-Madsen, O. Boric-Lubecke, and V. Lubecke, "Detection of multiple heartbeats using Doppler radar," in Proc. IEEE Int. Acoust., Speech, Signal Process. Conf., Toulouse, France, May 2006, pp. II-1160-II-1163.

[5] N. Petrochilos, M. Rezk, A. Host-Madsen, V. Lubecke, and O. Boric-Lubecke, "Blind seperation of human heartbeats and respiration by the use of a Doppler radar remote sensing," in Proc. IEEE Int. Acoust., Speech, Signal Process. Conf., Honolulu, HI, Apr. 2007, pp. I-333-I-336.

[6] M. I. Skolnik, "CW and frequency-modulated radar," in Introduction to Radar System, 3rd ed. New York: McGraw-Hill, 2001, pp. 68-100.

[7] R. J. Fontana, "Recent system applications of short-pulse ultra-wideband (UWB) technology," IEEE Trans. Microw. Theory Techn., vol. 52, no. 9, pp. 2087-2104, Sep. 2004.

[8] C. Li and J. Lin, "Recent advances in Doppler radar sensors for pervasive healthcare monitoring," in Proc. Asia-Pacific Microw. Conf., Yokohama, Japan, Dec. 2010, pp. 283-290.

[9] P. D. L. Beasley, "The influence of transmitter phase noise on FMCW radar performance," in Proc. 3rd Eur. Radar Conf., Manchester, U.K., Sep. 2006, pp. 1810-1813.
[10] S. Salous, "IF digital generation of FMCW waveforms for wideband channel characterization," Proc. Inst. Elect. Eng. I-Commun. Speech. Vis., vol. 139, no. 2, pp. 281-288, Jun. 1992.

[11] C. Wagner, A. Stelzer, and H. Jager, "PLL architecture for 77-GHz FMCW radar systems with highly-linear ultra-wideband frequency sweeps," in IEEE MTT-S Int. Microw. Symp. Dig., San Francisco, CA, Jun. 2006, pp. 399-402.

[12] S. Scheiblhofer, S. Schuster, and A. Stelzer, "High-speed FMCW radar frequency synthesizer with DDS based linearization," IEEE Microw. Wireless Compon. Lett., vol. 17, no. 5, pp. 397-399, May 2007.

[13] J. Lee, Y.-A. Li, M.-H. Hung, and S.-J. Huang, "A fully-integrated 77-GHz FMCW radar transceiver in 65-nm CMOS technology," IEEE J. Solid-State Circuits, vol. 45, no. 8, pp. 1542-1553, Aug. 2010.

[14] P. D. L. Beasley, A. G. Stove, B. J. Reits, and B. As, "Solving the problem of a single antenna frequency modulated CW radar," in Proc. IEEE Int. Radar Conf., Arlington, VA, May 1990, pp. 391-395.

[15] C. Metz, J. Grubert, J. Heyen, A. F. Jacob, S. Janot, E. Lissel, G. Oberschmidt, and C. Stange, "Fully integrated automotive radar sensor with versatile resolution," IEEE Trans. Microw. Theory Techn., vol. 49, no. 12, pp. 2560-2566, Dec. 2011.

[16] V. Z. Viskanta and R. E. Rose, "Conical scanning system for pioneer jupiter spacecraft,” IEEE Trans. Areosp. Electron. Syst., vol. AES-8, no. 2, pp. 236-245, Mar. 1972.

[17] A. D. Seifer, "Monopulse-radar angle tracking in noise or noise jamming," IEEE Trans. Areosp. Electron. Syst., vol. 28, no. 3, pp. 622-638, Jul. 1992.

[18] W. M. Brown, "Synthetic aperture radar," IEEE Trans. Areosp. Electron. Syst., vol. AES-3, no. 2, pp. 217-229, Mar. 1967.

[19] D. S. Goshi, Y. Liu, K. Mai, L. Bui, and Y. Shih, "Recent advances in $94 \mathrm{GHz}$ FMCW imaging radar development," in IEEE MTT-S Int. Microw. Symp. Dig., Boston, MA, Jun. 2009, pp. 77-80.

[20] G. L. Charvat, L. C. Kempel, E. J. Rothwell, C. M. Coleman, and E. L. Mokole, "A through-dielectric radar imaging system," IEEE Trans. Antennas Propag., vol. 58, no. 8, pp. 2594-2603, Aug. 2010.

[21] T. S. Ralston, G. L. Charvat, and J. E. Peabody, "Real-time throughwall imaging using an ultrawideband multiple-input multiple-output (MIMO) phased array radar system," in IEEE Int. Phased Array Syst. Technol. Symp. Dig., Waltham, MA, Oct. 2010, pp. 551-558.

[22] A. G. Stove, "Linear FMCW radar techniques," Proc. Inst. Elect. Eng. F-Radar Signal Process., vol. 139, no. 5, pp. 343-350, Oct. 1992.

[23] J. Geisheimer and E. F. Greneker, III, "Remote detection of deception using radar vital signs monitor technology," in Proc. IEEE Int. Carnahan Security Technol. Conf., Ottawa, ON, Canada, Oct. 2000, pp. 170-173.

[24] O. Postolache and R. N. Madeira, "Microwave FMCW Doppler radar implementation for in-house pervasive health care system," in IEEE Int. Med. Meas. Appl. Process Workshop, Ottawa, ON, Canada, May 2010, pp. 47-52.

[25] F.-K. Wang, C.-J. Li, C.-H. Hsiao, T.-S. Horng, J. Lin, K.-C. Peng, J.-K. Jau, J.-Y. Li, and C.-C. Chen, "A novel vital-sign sensor based on a self-injection-locked oscillator," IEEE Trans. Microw. Theory Techn., vol. 58 , no. 12 , pp. 4112-4120, Dec. 2010.

[26] F.-K. Wang, C.-J. Li, C.-H. Hsiao, T.-S. Horng, J. Lin, K.-C. Peng, J.-K. Jau, J.-Y. Li, and C.-C. Chen, "Single-antenna Doppler radars using self and mutual injection locking for vital sign detection with random body movement cancellation," IEEE Trans. Microw. Theory Techn., vol. 59, no. 12, pp. 3577-3587, Dec. 2011.

[27] F.-K. Wang, T.-S. Horng, K.-C. Peng, J.-K. Jau, J.-Y. Li, and C.-C. Chen, "Seeing through walls with a self-injection-locked radar to detect hidden people," in IEEE MTT-S Int. Microw. Symp. Dig., Montreal, QC, Canada, Jun. 2012, pp. 1-3, Sec. THPE-4.

[28] W. L. Stutzman and G. A. Thiele, "Aperture antennas," in Antenna Theory and Design, 1st ed. Toronto, ON, Canada: Wiley, 1981, pp. 375-445.

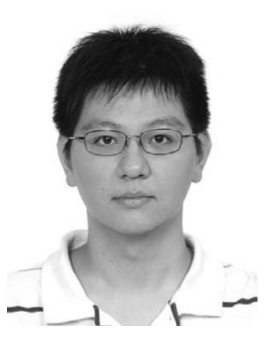

Fu-Kang Wang (S'10) was born in Kaohsiung, Taiwan, on May 15, 1985. He received the B.S.E.E. and M.S.E.E. degrees from National Sun Yat-Sen University, Kaohsiung, Taiwan, in 2007 and 2009, respectively, and is currently working toward the $\mathrm{Ph} . \mathrm{D}$. degree in electrical engineering at National Sun Yat-Sen University. His doctoral research is focused on RF sensing techniques. 


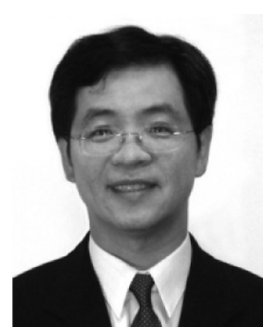

Tzyy-Sheng Horng (S'88-M'92-SM'05) was born in Taichung, Taiwan, on December 7, 1963. $\mathrm{He}$ received the B.S.E.E. degree from National Taiwan University, Taipei, Taiwan, in 1985, and the M.S.E.E. and Ph.D. degrees from the University of California at Los Angeles (UCLA), in 1990 and 1992, respectively.

Since August 1992, he has been with the Department of Electrical Engineering, National Sun Yat-Sen University, Kaohsiung, Taiwan, where he was the Director of the Telecommunication Research and Development Center (2003-2008) and Director of the Institute of Communications Engineering (2004-2007), and where he is currently a Distinguished Professor. He has authored or coauthored over 200 technical publications published in refereed journals and conferences proceedings, mostly in IEEE publications. He holds over ten patents. His research interests include RF and microwave integrated circuits (ICs) and components, RF signal integrity for wireless system-in-package, digitally assisted RF technologies, and green radios for cognitive sensors and Doppler radars.

Dr. Horng has served on several Technical Program Committees of international conferences including the International Association of Science and Technology for Development (IASTED) International Conference on Wireless and Optical Communications, the IEEE Region 10 International Technical Conference, the IEEE International Workshop on Electrical Design of Advanced Packaging and Systems (EDAPS), the Asia-Pacific Microwave Conference (APMC), the IEEE Radio and Wireless Symposium (RWS), and the Electronic Components and Technology Conference (ECTC). He has also served on the Project Review Board in the Programs of Communications Engineering and Microelectronics Engineering, National Science Council, Taiwan. $\mathrm{He}$ is the founder chair of the IEEE Microwave Theory and Techniques Society (IEEE MTT-S) Tainan Chapter. He is currently an associate editor for the IEEE TRANSACTIONS ON MiCROWAVE THEORY AND TECHNIQUES. He is a member of the IEEE MTT-S Technical Committee MTT-10 and MTT-20. He was the recipient of the 1996 Young Scientist Award presented by the International Union of Radio Science, the 1998 Industry-Education Cooperation Award presented by the Ministry of Education, Taiwan, and the 2010 Distinguished Electrical Engineer Award presented by the Chinese Institute of Electrical Engineering, Kaohsiung Branch, Taiwan. He was also the recipient of the 2011 Advanced Semiconductor Engineering (ASE) Inc. Chair Professorship and the 2012 Outstanding Research Award of National Sun Yat-Sen University.

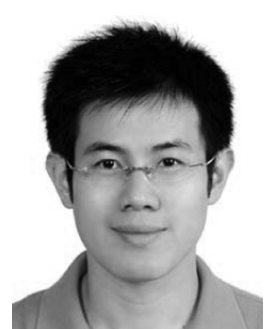

Kang-Chun Peng (S'00-M'05) was born in Taipei, Taiwan, on February 18, 1976. He received the B.S.E.E., M.S.E.E., and Ph.D. degrees from National Sun Yat-Sen University, Kaohsiung, Taiwan, in 1998, 2000 and 2005, respectively.

$\mathrm{He}$ is currently an Assistant Professor with the Department of Computer and Communication Engineering, National Kaohsiung First University of Science and Technology, Kaohsiung, Taiwan. His current research interests are in the area of delta-sigma modulation techniques, low-noise PLLs, low-power VCOs, and modulated frequency synthesizers.

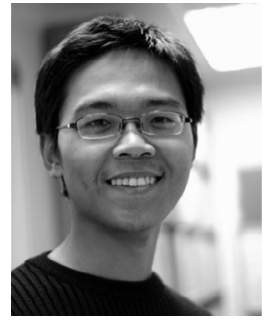

Je-Kuan Jau was born in Tainan, Taiwan, on November 19, 1977. He received the B.S.E.E. and Ph.D. degrees from National Sun Yat-Sen University, Kaohsiung, Taiwan, in 1999 and 2006, respectively.

$\mathrm{He}$ is currently with the Information and Communications Research Laboratories, Industrial Technology and Research Institute (ITRI), Hsinchu, Taiwan, as an Engineer. His research interests are RF transmitters and PAs.

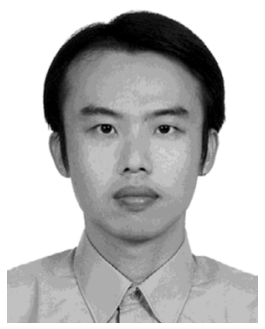

Jian-Yu Li (S'07) was born in Pingtung, Taiwan. He received the B.S.E.E and M.S.E.E degrees from National Sun Yat-Sen University, Kaohsiung, Taiwan, in 1998 and 2000, respectively, and is currently working toward the Ph.D. degree in communication engineering at National Chiao Tung University, Hsinchu, Taiwan.

In 2000, he joined the Information and Communications Research Laboratories, Industrial Technology and Research Institute (ITRI), Hsinchu, Taiwan, as an Engineer. His research interests are in the area of microwave integrated circuits and passive components design.

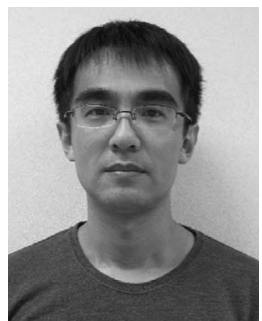

sign.
Cheng-Chung Chen was born in Hsinchu, Taiwan, in 1975 . He received the B.S. degree in electrical engineering from National Sun Yet-Sen University, Kaoshiung, Taiwan in 1997, and the M.S. and $\mathrm{Ph} . \mathrm{D}$. degrees in communication engineering from National Chiao-Tung University, Hsinchu, Taiwan, in 1999 and 2003, respectively.

In 2003, he joined the Information and Communications Research Laboratory, Industrial Technology and Research Institute (ITRI), Taiwan. His research interests include RF front-end chip and module de- 